\title{
Nanoparticles: augmenting tumor antigen presentation for vaccine and immunotherapy treatments of cancer
}

\author{
Charles B Chesson ${ }^{1,2}$ \& Andrew Zloza*,1,2 \\ ${ }^{1}$ Section of Surgical Oncology Research, Division of Surgical Oncology, Rutgers Cancer Institute of New Jersey, New Brunswick, NJ \\ 08903, USA \\ ${ }^{2}$ Department of Surgery, Rutgers Robert Wood Johnson Medical School, The State University of New Jersey, New Brunswick, NJ \\ 08903, USA \\ * Author for correspondence: andrew.zloza@rutgers.edu
}

The major goal of immunity is maintaining host survival. Toward this, immune cells recognize and eliminate targets that pose a danger. Primarily, these are external invaders (pathogens) and internal invaders (cancers). Their recognition relies on distinguishing foreign components (antigens) from self-antigens. Since cancer cells are the host's own cells that are harmfully altered, they are difficult to distinguish from normal self. Furthermore, the antigens least resembling the host are often sequestered in parts of the tumor least accessible to immune responses. Therefore, to sufficiently boost immunity, these tumor antigens must be exposed to the immune system. Toward this, nanoparticles provide an innovating means of tumor antigen presentation and are destined to become an integral part of cancer immunotherapy.

First draft submitted: 9 August 2017; Accepted for publication: 18 October 2017; Published online: 3 November 2017

Keywords: cancer $\bullet$ immunotherapy $\bullet$ nanoparticles $\bullet$ tumor antigens $\bullet$ vaccines

\section{Plain language summary}

Since cancer cells are derived from normal cells that have become altered, they are difficult for the immune system to recognize and eliminate. However, presentation of altered pieces of cancer cells on nanoparticles provides an innovating way to boost anticancer immune responses.

The ultimate success of cancer therapy lies in harnessing the power of the body's natural immune defenses. Proper activation of those defenses can prevent and eliminate growth, metastasis and recurrence of tumors [1-3]. While the field of cancer immunotherapy has exploded with a range of immunomodulatory agents (from passive agents like cytokines and checkpoint inhibiting antibodies, to active agents like adoptively transferred altered $\mathrm{T}$ cells and antigen-presenting cell [APC] vaccines), only a small proportion of treated patients have experienced long-term benefit. Although in some ways we have come a long way in advancing our basic tumor immunology and cancer biology knowledgebase, and in translating that to clinical trials and patient care regimens, in other ways we are still at the infancy of our discovery process. Therefore, rather than working on limited understanding and assumptions, the most immediate and effective advances may come from focusing on boosting the natural immune defenses against cancer, even if we do not yet understand all the underlying mechanisms involved.

Many solid tumors are recognized and eliminated by active immune surveillance through cytotoxic lymphocytes (i.e., CD8 ${ }^{+} \mathrm{T}$ cells and natural killer $[\mathrm{NK}]$ cells) $[4,5]$. As tumors progress in size and severity, their microenvironments become increasingly immunosuppressive. Natural surveillance becomes compromised and lymphocytes within the tumor can indirectly lead to tumor growth through the secretion of immune-suppressive cytokines (e.g., TGF- $\beta$ and IL-10) [6,7]. Active immunotherapies provide necessary immune triggers that drive the expansion of tumor-infiltrating $\mathrm{CD}^{+} \mathrm{T}$ cells leading to tumor reduction and eradication. Activation and expansion of tumor-infiltrating lymphocytes begins with the delivery of tumor-associated antigens (TAAs) to APCs and the presentation of such antigens in the context of MHC class I molecules (for in-depth reviews of antigen-processing and presentation of antigens, see $[8,9])$. Traditional APCs require activation signals to fully mature and migrate to 
the draining lymph nodes where they can present antigen to naive $\mathrm{T}$ cells. However, antigen presentation likewise occurs within the tumor microenvironment and by a variety of cells including B cells. Recognition of an MHC-TAA complex by a $\mathrm{T}$ cell with the cognate $\mathrm{T}$-cell receptor (signal 1) along with proper costimulation (signal 2) and cytokine signaling (signal 3) drives the expansion of tumor-specific T lymphocytes. If primed within the lymph nodes, these lymphocytes must then migrate to the tumor to eliminate the primary cancer and throughout the body to eliminate metastases.

Antigens present within the tumor are mainly self-antigens to which cognate $\mathrm{T}$ cells have been eliminated by central tolerance in the thymus during their development or suppressed outside of the thymus by peripheral tolerance. Therefore, the tumor antigens that can be recognized and to which the immune system can respond are self-antigens that have been mutated [10]. Identification of these antigens for vaccine development and cancer treatment can be complex and costly. This is in large part due to a current lack of knowledge as to which identified mutated antigens should be targeted. Targeting all mutated antigens that can be identified is not feasible with technologies currently utilized for this purpose (although this idea will be revisited in the discussion of the advantages of utilizing nanoparticles). Targeting random-mutated antigens may result in the selection of antigens that yield limited immune responses [11-13]. Conversely, targeting a select group of antigens that yield high in vitro immune responses may result in redundant attempts to activate cognate $\mathrm{T}$ cells that are already engaged and whose additional stimulation contributes little further antitumor response.

Alternatively, tumor lysates and whole-cell tumor preparations with self-antigens provide a more complete antigenic profile of the tumor and obviate the need to define, select and synthesize TAA peptide epitopes [14,15]. However, whole-cell tumor vaccines have to-date exhibited poor immunogenicity when administered alone, most likely due to their inability to induce dendritic cell (DC) maturation and their propensity to promote immune tolerance rather than activation [16]. Preparation methods of both tumor lysate and whole-cell tumor vaccines will be critical for optimizing their immunogenicity and effectiveness in the future.

The passive administration of inhibitory receptor (immune 'checkpoint') blockade treatments, including antiPD-1, anti-PD-L1 and anti-CTLA-4 has been US FDA-approved and has revolutionized the treatment and prognosis of late-stage melanoma, lung and renal cancers [17,18]. Unfortunately, this approach is limited to those patients with preexisting levels of antitumor $\mathrm{T}$ cells within their tumors and even among these only a small proportion of checkpoint inhibitor-treated patients exhibit long-term benefit. Active immunotherapies that are highly efficacious in the delivery and presentation of TAAs to the tumor microenvironment are expected to improve outcomes for cancer patients by recruiting immune responses to the tumor site and providing the necessary stimulus for tumor elimination. This highlights the need to develop novel techniques and strategies that efficiently deliver TAAs to APCs, promote TAA presentation and the activation of the APCs, and lead to improved clinical outcomes with the goal of tumor eradication and prevention. Studies that highlight the different delivery platforms and strategies used to promote enhanced antigen presentation and downstream lymphocyte activation in cancer models are highlighted in Table 1. Nanoscale technologies are promising avenues for active cancer immunotherapies and particle-based platforms have been used for effective delivery of DNA, siRNA and protein antigens as well as a plethora of adjuvants and immunomodulatory compounds. Current research into nanoscale platforms for active immunotherapy aims to optimize the combination of targeting antigens and tethering adjuvants for optimal antigen presentation and downstream activation of $\mathrm{T}$ lymphocytes.

\section{Nanoparticle antigen delivery platforms \& beyond}

Nanoscale applications for antigen packaging and delivery have become ubiquitous in the field in recent years. Nanoparticles are carrier platforms that can encapsulate or surface incorporate both adjuvant and antigen without initiating an immune response to the carrier. For an excellent review on the different types of materials and designs of nanoparticles for cancer as well as their use in human trials and toxicity, please see [19,20]. Nanoparticle carriers are an efficient means by which to deliver TAAs and immunomodulatory molecules to APCs in order to propagate T-cell activation, expansion and response (Figure 1A). Soluble delivery of TAAs and tumor lysates is subject to rapid clearance, and these are poorly taken up by APCs. Surface conjugation or encapsulation of whole protein or peptide antigens onto particulates can facilitate antigen uptake, cross-presentation (CP) and APC maturation. Several studies utilizing the chicken egg protein, OVA or the $\mathrm{CD}^{+}{ }^{+}$-cell epitope $\mathrm{OVA}_{(257-264)}$ conjugated to the surface of gold solid-core particles [21], polystyrene beads [22] or encapsulin nanoparticles [23] have shown protective prophylactic as well as therapeutic immunity to B16 melanoma expressing OVA and EL4 lymphoma-expressing OVA. However, these tumors express OVA, which is a nonself (i.e., a foreign) antigen. Since patient tumors do 


\begin{tabular}{|c|c|c|c|}
\hline $\begin{array}{l}\text { Strategy to enhance antigen } \\
\text { presentation or immunogenicity }\end{array}$ & Type & Tumor models & Mechanism of antigen enhancement \\
\hline VLPs and particulate delivery & $\begin{array}{l}\text { Viral mimics, exosomes [33-36], } \\
\text { nanoparticulate delivery [38] }\end{array}$ & Breast, B16 melanoma, B16-OVA & $\begin{array}{l}\text { Increased uptake and cross-presentation } \\
\text { within MHC class I }\end{array}$ \\
\hline Endosomal antigen escape & pH responsive polymers [39-45] & OVA, B16-OVA & Improved antigen loading onto $\mathrm{MHC}$ \\
\hline Production of ROS & $\begin{array}{l}\text { Lysosomal rupture, trans-RA, cationic } \\
\text { liposomes [46-49] }\end{array}$ & B16 & $\begin{array}{l}\text { Activation of APCs and increased MHC } \\
\text { expression }\end{array}$ \\
\hline Protein modification: oxidation & $\mathrm{HOCl}$ cell treatment [59-61] & B16-OVA, prostate, ovarian & $\begin{array}{l}\text { Aldehyde-modified side chains promote } \\
\text { uptake and presentation }\end{array}$ \\
\hline Protein modification: heat shock & High-temperature incubation $[62,63]$ & Glioblastoma & $\begin{array}{l}\text { Increased expression of heat-shock } \\
\text { proteins }\end{array}$ \\
\hline TLR agonists and adjuvant delivery & $\begin{array}{l}\text { TLR2 }[91], \text { TLR4 }[81,92,93], \\
\text { TLR7/8 [85-87], TLR9 }[82-86,90] \text {, } \\
\text { complement proteins }[97], \\
\text { cytokines }[100,101]\end{array}$ & Breast, ovarian, prostate & $\begin{array}{l}\text { Activation and maturation of APCs, } \\
\text { cytokine expression }\end{array}$ \\
\hline Physical size of delivery platform & $<200 \mathrm{~nm}, 200-800 \mathrm{~nm}, 1.0 \mu \mathrm{m}+[18,37]$ & B16-OVA. HPV/cervical & $\begin{array}{l}\text { Efficient trafficking to lymph nodes and } \\
\text { uptake by APCs }\end{array}$ \\
\hline
\end{tabular}

not express such foreign antigens, findings from models utilizing these antigens may not be extrapolated to real-life scenarios. Such models are also inherently prone to loss of OVA antigen presentation that renders them hidden from OVA antigen-specific $T$ cells and thus using these models to track antigen presentation or T-cells responding to these antigens is problematic [24]. Tumor lysate rendered from tumor cell lines or disruption of excised solid tumors can provide a more complete antigenic profile and limit erroneous outcomes due to expression of foreign antigens. Our group has shown promising results from studies utilizing a single-step nanoparticle antigen-presenting system, which are made from the tethering of tumor lysate as the source of antigen. Without the addition of any exogenous adjuvant, these particles significantly prevent tumor growth when administered prophylactically and result in increased APC and tumor-specific $\mathrm{CD}^{+}$T-cell infiltration within the tumor microenvironment [25]. Another promising avenue for the delivery of broader repertoires of TAAs includes the coating of intact tumor cell membranes and surface proteins on poly(latic-co-glycolic acid) (PLGA) polymeric particles [26]. Here, cellular membranes are derived from B16 and MDA-MB-435 melanoma cell lines and the particles subsequently modified with the adjuvant monophosphoryl lipid A, a Toll-like receptor (TLR) 4 agonist. This promising technology induces strong expression of costimulatory molecules CD40, CD80 and CD86 on CD11c ${ }^{+}$DCs; however, it remains unknown whether the reported upregulation of costimulatory DC markers and antigen presentation results in physiologic antitumor in vivo effects [26].

Virus-like particles (VLPs) resemble the size and architecture of viruses. VLPs can be surface-modified with TAAs and they are typically 10-100 $\mathrm{nm}$ in diameter, which allows efficient uptake from DCs and trafficking to draining lymph nodes [27]. VLP studies with TAAs have had mixed success. In a study with bovine papillomavirus expressing the common ductal adenocarcinoma MUC1 epitope, a VLP-based vaccine induced strong APC costimulatory molecule expression and delayed the growth of tumor, but ultimately provided minimal protection [28]. Similar results have been published with rabbit hemorrhagic disease virus with surface-conjugated OVA. Here, delay of B16-OVA tumor growth after vaccination was achieved, although this resulted in minimal overall protection after tumor progression [29]. Although more research is needed on VLP delivery vehicles for antitumor responses, the first VLP vaccines have recently been approved for the prevention of the oncogenic HPV and are, therefore, preventive for cervical carcinomas and other HPV-associated mucosal carcinomas in men and women [30,31].

Alternatively, virosomes are a promising vector for tumor antigen delivery. Virosomes mimic native viral structures through the inclusion of purified viral envelope proteins into a lipid bilayer lacking any viral genomic material of the native virus [32]. For example, influenza virosomes can be formed from purified hemagglutinin and neuraminidase envelope proteins incorporated into a mixture of synthetic phosphatidylcholine and phosphatidylethanolamine lipids, as well as natural viral lipids [33]. Immunopotentiating reconstituted influenza virosomes are the most extensively studied viral liposomes and these can serve as carriers for multiple antigens [34]. Encapsulation of peptide antigens inside the liposomal vectors has been shown to be an effective strategy to elicit cellular immune responses directed against melanoma and breast cancer TAAs in animal studies [32]. A Phase I clinical trial of influenza virosomes encapsulated with Her/neu peptide antigen was well tolerated by ten patients with metastatic breast 
(A)

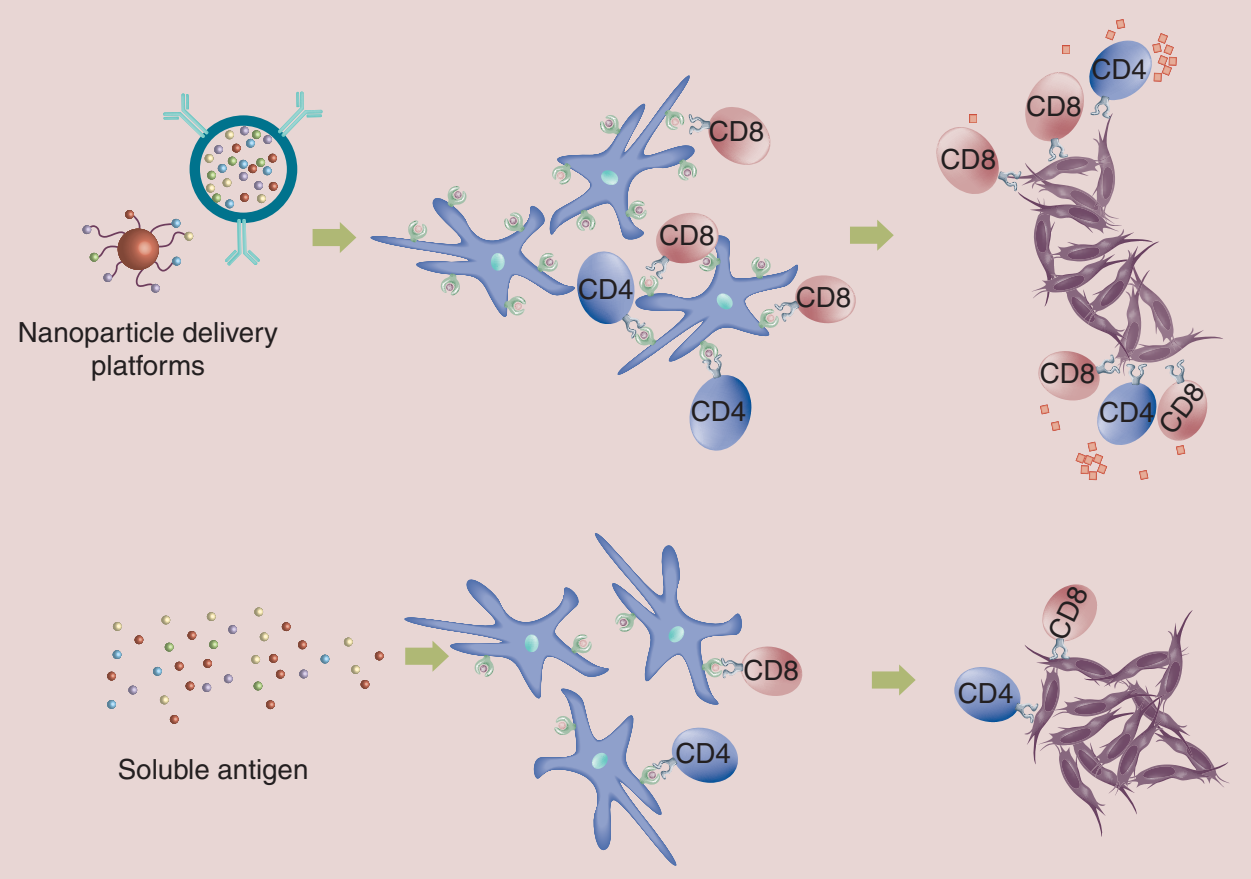

(B)
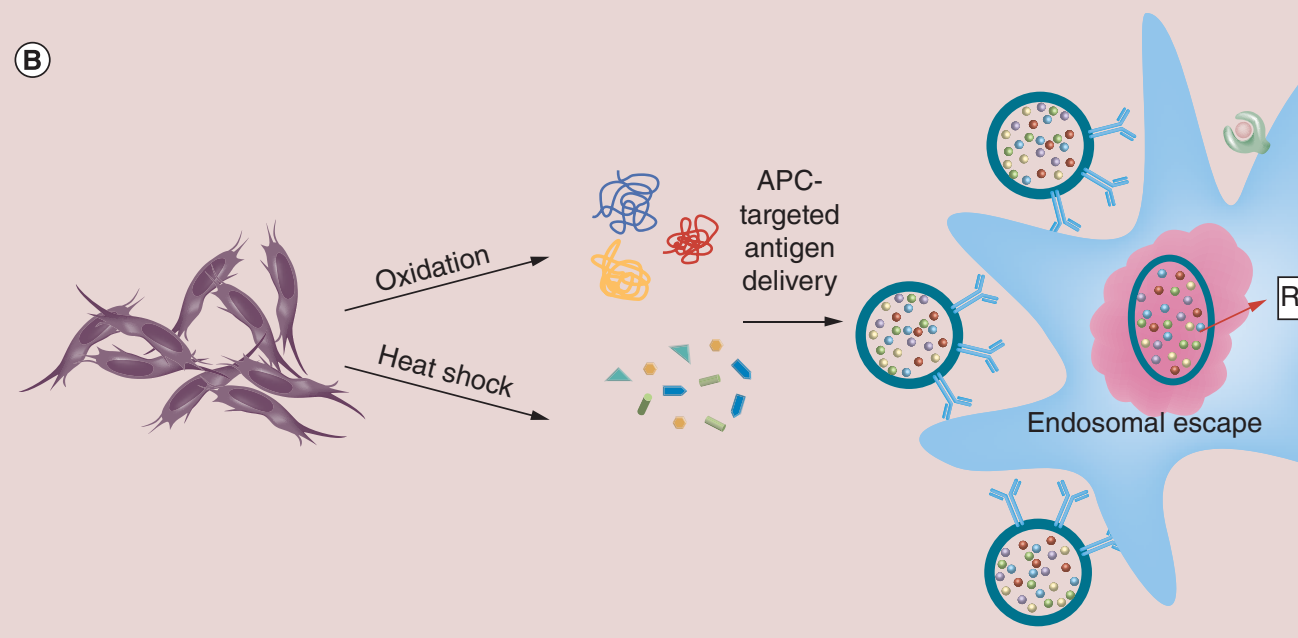

Figure 1. Methods for enhancing antigen uptake and presentation for cancer immunotherapies and vaccines. (A) Tumor antigen delivery via nanoparticles creates APC antigen depots and increases antigen presentation. Nanoscale platforms may be used in both settings to increase antigen uptake and presentation by MHC class I and II molecules, subsequently activating both CD8 ${ }^{+}$and CD4 ${ }^{+} \mathrm{T}$ cells for antitumor immunity, respectively. (B) Protein modification through oxidation or heat shock enhances antigen uptake and as well as APC activation. Antigens taken up by APCs that escape the endosome are more efficiently cross-presented. This may activate ROS, thus driving activation of APCs. Exogenous and codelivered TLR agonists as well as DAMPs and ligands for pathogen-activated recognition receptors contribute to APC activation and maturation, which can then upregulate costimulatory molecules and cytokine expression. APC: Antigen-presenting cell; DAMP: Damage-associated molecular pattern; ROS: Reactive oxygen species; TLR: Toll-like receptor.

cancer. These virosomes demonstrated both humoral and cellular immune responses to the peptide antigens with a notable decrease in circulating $\mathrm{CD} 4^{+} \mathrm{CD} 25^{+} \mathrm{Foxp} 3^{+}$Treg cells [35]. Although larger clinical trials are needed to show efficacy, significant preclinical evidence in animals suggests that virosomes as vaccine modalities can initiate tumor antigen-specific cell-mediated immunity and may prove to be strong candidates for future cancer immunotherapies $[36,37]$. 
Tumor-derived exosomes may be a potential alternative to using tumor lysate for multivalent tumor antigen delivery. Exosomes range from 30 to $100 \mathrm{~nm}$ in size and are secreted as intracellular vesicles that fuse with the plasma membrane and contain a mixture of both cellular cytosolic and membrane proteins as well as mRNA [38]. Exosomes derived from DCs may contain complexes of MHC and costimulatory molecules while those derived from tumor cells may encompass a representative sample of TAAs. Within the tumor microenvironment, tumor cell exosomes may be inherently immunosuppressive by encompassing the same repertoire of tumor suppressive ligands and receptors, such as Fas-L and HLA-G, or by inducing immune tolerance [38]. Therefore, preparation of tumor-derived exosomes may require additional adjuvants, cytokines or cell-line treatments prior to purification [39]. Subjecting A20 lymphoma cells to heat shock has been shown to increase the levels of HSP60 and HSP90 found within the exosomes, as well as MHC-I, costimulatory molecules such as CD40, and inflammatory cytokines. Four doses of HS exosomes were observed to protect $90 \%$ of animals for 40 days while therapeutic administration starting 1 week after A20 challenge resulted in decreased tumor growth and 40\% survival [40]. Alternatively, DCs given appropriate maturation signals can be induced to release exosomes that can then be harvested and pulsed with selected antigens for vaccination. DC exosomes containing OVA have been shown to be protective in therapeutic melanoma models when combined with the TLR3 agonist poly(I:C) resulting in 60\% survival [41]. Enhanced protection was due in part to the recruitment of NK and natural killer T (NKT) cells to the tumor site. However, again, these findings require validation in similar studies not utilizing a foreign antigen, such as OVA.

\section{Physical determinants important for antigen presentation}

Particle size is a critical factor for determining localization within the tissue and uptake by cell type. Larger particles, greater than $200 \mathrm{~nm}$ are typically retained at the site of inoculation while smaller particles under 100-nm traffic efficiently to the draining lymph nodes and are taken up by DCs [42]. DCs are undoubtedly efficient at $\mathrm{CP}$ of exogenous antigen and are, therefore, targeted for antigen delivery. A comparative study on particle size demonstrated that 40 -nm PS particles were the most efficient immunostimulatory antigen-delivery particles among those ranging in size from $20 \mathrm{~nm}$ to $2 \mu \mathrm{m}$ [22]. Prophylactic vaccination with two doses of 40-nm PS-OVA particles without extraneous adjuvants prevented EG7-OVA tumor growth in 100\% of animals until day 10 while tumor growth in groups administered 1.0- $\mu \mathrm{m}$ PS-OVA particles was comparable to that of control groups. Therapeutic challenges were only performed with 40-nm particles using the EG7-OVA model, but these studies also showed that such particles are effective in reducing tumor size or inhibiting further growth when administered on 8 days after tumor challenge in mice. However, variables beyond the size of the particles such as type of antigen and concentration, tumor model, injection site and time of inoculation must be considered in the overall design of tumor vaccines and immunostimulants.

$\mathrm{CP}$ is a process by which APCs take up, process and present extracellular antigens within MHC class I molecules to $\mathrm{CD}^{+} \mathrm{T}$ cells. In order for $\mathrm{CP}$ of such exogenous antigens to occur, the antigen must escape the acidic environment of the endo/lysosome into the cytosol where it is processed through the proteasome machinery for MHC-I antigen presentation [43]. PLGA particles efficiently release their payload from the endosome into the cytosol where they can be ushered into the class I presentation pathway. Polymers, chemistries and cross-linkers that are $\mathrm{pH}$ sensitive may also facilitate release of antigen payloads from the endosome [44,45]. Polyacrylamide nanoparticles with functionalized $\mathrm{pH}$-sensitive cross-linkers have been observed to be more effective at presenting antigen compared with nonacid degradable particles, which are compounded by increased endocytosis due to the incorporation of cationic polymers that increase the surface charge [46]. Acid degradable particles loaded with OVA antigen have been shown to result in a potent antitumor response with 50\% survival of hosts for 30 days, although studies comparing these to nonacid degradable particles are needed [47]. Alternatively, a novel platform has been described that promotes antigen release by encapsulating $\mathrm{NH}_{4} \mathrm{HCO}_{3}$ into PLGA particles. The ammonium bicarbonate ions balance the $\mathrm{pH}$ within the endosome and produce $\mathrm{CO}_{2}$ and $\mathrm{NH}_{3}$, which disrupt the nanoparticle shell [48]. Reductive conditions within the endosome can also be exploited to facilitate antigen release when antigens are conjugated to carriers through disulfide linkages [49]. Polypropylene sulfide-modified particles with conjugated OVA via a reducible disulfide linkage are more efficiently cross-presented in MHC-I molecules than OVA conjugated through nonreducible bonds, resulting in significantly higher expansion of effector and memory tumor antigenspecific $\mathrm{CD}^{+} \mathrm{T}$ cells [50]. These findings highlight promising chemical and biochemical strategies for enhancing $\mathrm{CP}$ of antigens, which may have a role in TAA delivery in future immunotherapies.

Lysosome rupture results in reactive oxygen species (ROS) that enhance DC maturation leading to efficient antigen CP [51]. Inducible ROS may, therefore, be a useful indirect adjuvant for cancer vaccine platforms (Figure 1B). 
Nanoparticles synthesized with a $\mathrm{pH}$-sensitive polysaccharide that incorporates transretinoic acid, a potent activator of ROS in DCs, result in robust activation of the proteasome and significantly increased MHC-I OVA presentation in mice [52]. Tumor progression is significantly decreased after three vaccinations and survival is prolonged. Cationic liposomes are also potent inducers of ROS [53,54]. Increasing molar quantities of cationic liposomes (DOTAP) in a dose-dependent fashion generates increasing amounts of ROS with contaminant negative feedback for antitumor immune responses, most likely due to an increase in the numbers of apoptotic cells. Optimal liposomal formulations that include neutral and cationic lipids induce an appropriate ROS in DCs in the absence of apoptosis, and have been shown to be effective in controlling tumor growth [55].

\section{Beyond nanoparticles: ex vivo DC therapies}

In 2010, the FDA approved the cancer vaccine, Sipuleucel-T (Provenge) for prostate cancer [56]. Sipuleucel-T is a vaccine made from patient-isolated DCs pulsed with known prostate TAAs and treated with immune cellmaturation cytokine, GM-CSF [57]. DC-based treatments, although effective, are high-cost modalities and the use of peptide TAAs limits their use to specific tumor profiles, thus requiring knowledge of TAAs and the patient HLA type. Pulsing DCs with a soluble patient-derived autologous tumor lysate broadens the antigen profile available for immune activation but suffers from failure to induce proper maturation. This may possibly be a result of the presence of immunoregulatory cytokines in the lysate, which can promote immune tolerance $[58,59]$. In clinical trials, pulsing DCs with soluble tumor lysate has had mixed results, with response rates of approximately $40 \%$ for regimens that often administer ten or more doses [14,60].

Nanoparticle delivery platforms that encapsulate or surface absorb TAAs, ultimately promote DC uptake, $\mathrm{CP}$ and maturation, while protecting TAAs from proteolytic degradation. In a preclinical study, a comparison between soluble tumor lysate and PLGA-encapsulated lysate from patient-derived tumors demonstrated that antigen encapsulation leads to robust autologous T-cell IFN- $\gamma$ production and IL-10 downregulation [61]. Additional studies with TARA R182 ovarian cancer cell lysate encapsulated within PLGA nanoparticles have shown efficient antigen uptake and presentation by DCs to $\mathrm{CD}^{+} \mathrm{T}$ cells, resulting in IL-6, IL-1 $\beta$ and IFN- $\gamma$ production [62]. Although thus far data demonstrating effectiveness in tumor reduction or prevention using nanoparticle delivery for ex vivo-loaded DC vaccines are lacking, technical advances are expected to make clinical translation possible.

The preparation of ex vivo DC vaccines typically requires the addition of adjuvants, TLR agonists or DC maturation ligands. Soluble adjuvants are typically mixtures of microbial components that stimulate pathogenassociated molecular pattern receptors. Ex vivo manipulation of DCs provides the distinct advantage of direct administration of adjuvants and TLR ligands to drive robust DC maturation and CP of TAAs, perhaps obviating the need for particulate delivery vehicles ex vivo [63]. Moreover, DCs treated with TLRs ex vivo in the absence of any actual tumor antigens have also shown to provide some efficacy as immunotherapies [64]. Comparatively, the method of protein antigen preparation may dictate successful antigen uptake and presentation (Figure 1B). Treating tumor cells with $\mathrm{HOCl}$ has been shown to increase both tumor antigen uptake and overall immunogenicity. $\mathrm{HOCl}$ oxidation exposes immunogenic peptides and reacts with hydroxyamino acid side chains to yield aldehyde by-products, which may play a role in inflammation and phagocyte activation $[65,66]$. DCs pulsed with $\mathrm{HOCl}-$ oxidized tumor antigens from ovarian and breast cancer lysate have demonstrated that oxidized antigen promotes better presentation and expanded T-cell activation and results in controlled tumor growth in early-phase human studies [67]. Induction of heat-shock proteins among tumor cell lines is another potential tool for increasing total immunogenicity and CP by APCs containing tumor lysate antigens. Heat-shocked prostate preparations of tumor antigens compared with controls have demonstrated strong antitumor immune responses in melanoma and prostate models in vivo, as well as in human trials against glioblastoma [68,69]. These strategies are promising avenues for enhancing the immunogenic potential of any tumor lysate used as the source of antigen; however, the complexities of ex vivo-manipulated DC-based vaccines (including choice of DC subtypes, inclusion of pathogen-associated molecular patterns or other maturation signals, and use of delivery platforms) are important concerns for designing future ex vivo DC-based vaccines.

\section{Beyond nanoparticles: in vivo DC targeting}

In vivo DC targeting can be used to optimize the delivery of TAAs for cancer vaccines. Particle size $(<500 \mathrm{~nm}$ and ideally 20-50 $\mathrm{nm}$ ) and the route of administration also function to passively target particulate vaccines to the draining lymph nodes and into DCs [70]. In therapeutic tumor models, pluronic-stabilized propylene sulfide nanoparticles bearing the nontumor antigen OVA have been effective in reducing tumor burden only when the 
vaccine is administered into the ipsilateral tumor-draining lymph node [71]. Cell entry-based active targeting utilizes surface-modified nanoscale vesicles with DC surface receptor ligands or antibodies to promote internalization and direct delivery to the proper APC subtype [72]. Families of DC-targeting receptors include costimulatory molecules, C-type lectin receptors and MHC-II molecules [73]. Targeting DC-specific receptors helps create an antigen depot effect in target APCs and has been shown to enhance antigen presentation leading to protection [74]. The optimal choice of DC-targeting ligands for cancer vaccines is a current subject of debate. With respect to particulate delivery systems, in a comparative study of PLGA NPs decorated with surface antibodies to DEC-205, CD11c or CD40, there was no difference in the immunological in vivo response between the different targeting moieties [75]. DCs inherently sample antigens from the extracellular environment and arguably targeting DC-specific receptors may be unnecessary. From a practical standpoint, the addition of DC receptor targets adds to the cost and complexity of GMP production. However, studies using particulate delivery systems do highlight the benefit of targeting DC receptors CD40, mannose receptor, DC-SIGN and DEC-205 for reducing or controlling tumor burden [76-78]. Furthermore, specifically targeting DC subsets known for efficiently cross-presenting exogenous antigens, especially those with human equivalents from preclinical animal studies such as DC-SIGN or CD205 may be excellent avenues for clinical translational [79,80]. Targeting DC-SIGN is well characterized with optimal-binding glycans in place of large monoclonal antibodies and several studies have shown as much as 100 -fold increases in antigen (e.g., OVA and MART-1 epitope) presentation on both MHC-I and MHC-II [81,82]. Future studies are on-going to determine which subsets of DCs are optimal targets and how this impacts antitumor immunity.

\section{Codelivery of TLRs \& antigen combinations}

Codelivery of antigens and immunomodulatory compounds such as TLR agonists and danger-associated molecular pattern ligands with nanoscale platforms provides activation signals necessary for DCs to fully mature and downstream stimulate $\mathrm{T}$ cells and B cells, while limiting the severe toxicity seen in systemic delivery of free TLRs [83]. TLRs are expressed mainly on macrophages, B cells and DCs, but they are also expressed transiently on activated T cells and tumor cells [84]. Activated T cells upregulate the expression of TLRs, and TLR expression levels decrease with the contraction of effector cell subsets. TLR ligands can stimulate or inhibit downstream antitumor activity of adaptive immune cells depending on the specific innate receptor and the cell type on which it is expressed [85]. Although resting and naive T cells are unresponsive to TLR ligands, tumor-suppressed lymphocytes within the tumor microenvironment and draining lymph nodes may benefit from enhanced antitumor activity, immune cell survival and memory cell formation. Evidence from studies using single and combination TLR agonists as adjuvant therapies supports this idea [86-88]. In a recent study, YSK05 liposomes encapsulating cyclic-di-GMP as an adjuvant were successful in significantly preventing B16 melanoma seeding within the lung, likely due to the recruitment of NK cells [89,90]. Conversely, other studies have reported that signaling through TLRs in certain cancers can likewise be proinflammatory and immunosuppressive as well as proangiogenic [84,91]. Included adjuvants for the purposes of increasing cytotoxic T-cell activation and expansion generally focus on TLR agonists, although alternative adjuvants such as $\alpha$-galactosylceramide ( $\alpha$-GalCer) are notable due to their ability to stimulate both the innate and adaptive arms of the immune response [92]. $\alpha$-GalCer is presented on MHC class I-like CD1d molecules that bind with the invariant T-cell receptor found on NKT cells. Activated NKT cells secrete Th1-like cytokines that induce DC maturation and expression of costimulatory molecules. When codelivered with the self-antigen TRP2 encapsulated in cationic liposomes, the formulation has been shown to be highly efficacious in a therapeutic setting against B16 melanoma in mice [93]. Additionally, adjuvants like $\alpha$-GalCer may work additively with TLR agonists to promote antigen uptake and presentation [94].

Nanoparticle platforms may encapsulate the antigen with surface-absorbed or covalently bound danger-associated molecular patterns, or encapsulate the antigen and adjuvant. Studies with PLGA nanoparticles coencapsulating 7-acyl lipid A, a TLR4 agonist, with the melanoma self-antigen TRP2 have shown efficient tumor growth control when vaccinations are administered early after B16-F10 melanoma challenge [95]. A novel platform was recently reported using mesoporous silica rods, which upon injection in vivo self-assemble into scaffolds that facilitate immune cell recruitment [96,97]. These mesoporous silica rods are functionalized with GM-CSF and CpG, a TLR9 agonist, and prevent tumor growth after one dose, with $90 \%$ host survival of 30-day post-treatment. Similarly, an injectable nanogel derived from cholesteryl pullulan along with $\mathrm{CPG}$ in a mouse model of colon carcinoma has been shown to offer $100 \%$ protection in the prophylactic setting and to suppress tumor growth in the therapeutic setting [98]. CpG as well as TLR7/8 agonists have also been shown to reduce the frequency of myeloid-derived suppressor cells and Tregs in the tumor microenvironment, which result in a shift from an immunosuppressive 
to an immunostimulatory environment [99,100]. TLR codelivery may also have utility in ex vivo models of DC immunotherapy, including ex vivo delivery of PLGA-encapsulated STAT3 siRNA, the TLR7 agonist imiquimod and OVA antigen to DCs [101]. Such PLGA particles have been shown to be highly effective at preventing tumor growth after prophylactic vaccination. Interestingly, the antigen- and adjuvant-combined effect was significantly greater when they were delivered as separate particles [102].

A stark advantage of particulate delivery is the design of multifunctional vehicles that may contain multiple TLRs, which may additively or synergistically improve the overall immune response and outcomes [103]. MelQbG10, a viral-like protein nanoparticle vaccine that incorporates both imiquimod and CpG TLR agonists, is currently in Phase IIa human trials with promising safety and immunogenicity data from Phase I trials as a treatment for advanced-stage melanoma [104]. Other avenues for multifunctional nanoparticle approaches to cancer vaccines may incorporate in vivo DC targeting along with TLR agonists for efficient activation of specific DCs or other APCs. PLGA particles encapsulating Pam3CSK4, a TLR2 agonist, and Poly(I:C), a TLR3 agonist, and coated with anti-CD40 have been shown to efficiently prevent tumor growth in a B16-OVA model with 50\% survival 40-day post-tumor challenge [105]. Other adjuvant combinations such as 4-1BBL, an agonist for the 4-1BB receptor expressed constitutively on DCs, and the TLR4 ligand, monophosphoryl lipid A, have been demonstrated to have potent antitumor therapeutic effects in models of lung cancer [106,107]. 4-1BBL as a single adjuvant increases IL-2 production, increases antigen uptake and presentation and drives Th1 T-cell responses responsible for antitumor efficacy, thus making a strong case for its future development as a potent anticancer adjuvant [108]. Although several clinical trials of TLR-agonist drugs have been terminated due to systemic inflammatory side effects, targeted delivery can reduce the toxicity of systemic administration by accumulation of the agonist at the tumor site and the lower dosage requirements of a targeted approach.

TLRs may also be expressed on malignant cells limiting their use as adjuvants in certain cases. TLR7 expression on non-small-cell lung cancer cells is associated with a poor outcome [109]. TLR7 agonists in mice with TLR7expressing tumor cells lead to resistance to chemotherapy and tumor progression. TLR7 signaling on lung cancer cells leads to the expression of antiapoptotic protein, Bcl-2, and recruitment of myeloid-derived suppressor cells into the tumor environment, both of which increase tumor cell survival [110]. TLR expression on tumor cells has been associated with positive and negative outcomes and several in-depth reviews exist [84,85]. Screening for TLR expression to design personalized care may be a necessary step in the future; however, this also may provide strong justification for designing improved DC cell entry-based targeted therapies that deliver TLR adjuvants to appropriate immune cells regardless of TLR expression on the tumor. Furthermore, combined use of multiple TLRs or TLRs combined with inhibitory receptor blockade therapies, without the use of targeted delivery molecules, may override the negative aspects of any TLR signaling on the tumor itself.

Adjuvants beyond TLR agonists are also being explored as potential cancer immunotherapies. The complement system is a key innate immune defense mechanism responsible for recognizing danger signals and responding through a protein-signaling cascade that triggers proinflammatory signals and chemokines that attract neutrophils and macrophages. C5a is one such protein involved in the complement signaling cascade with adjuvant potential [111]. A study using a fusion protein from a TLR4 ligand, OVA antigen and complement protein, C5a, has reported that the fusion protein doubles survival against EG7.OVA tumor challenge after one dose of vaccine compared with treatment with or without C5a [112]. The concept of using ILs or IL receptors to direct and augment the immune response to vaccines is also being explored. IL-15 is an important cytokine involved in the regulation of cytotoxic $T$ cells and has shown promise in conjunction with DNA vaccines in enhancing antigen-specific T-cell responses [113]. IL-15 conjugated to PLGA nanoparticles with encapsulated OVA has been shown to efficiently activate antigen-specific $\mathrm{CD}{ }^{+} \mathrm{T}$ cells and significantly increase overall survival of hosts after tumor challenge [114]. IL-23, another proinflammatory cytokine has been shown to have potent adjuvant effects on vaccine-derived T cells by enhancing the effector function of $\mathrm{CD}^{+}$tumor-infiltrating lymphocytes and suppressing tumor growth [115]. This was most pronounced when IL-23 was expressed locally in the tumor and the side effects seen with systemic administration may have been the result of IL-23-induced IL-17 expression. Continued development of novel adjuvants and combinations of adjuvants for future cancer vaccines may be necessary for improved patient outcomes and may require personalized approaches defined by the tumor phenotype.

\section{Conclusion \& future perspective}

Enhancing the presentation of tumor-associated self-antigens or neoantigens on APCs is a critical step in driving a natural antitumor immune response but it is also a small step in the overall goal. In many tumors, functional antigen 
presentation is impaired or the expression of MHC is downregulated limiting the benefit of many antigen-specific anticancer vaccines. In other tumors where MHCs present altered self-antigens or TAAs, particulate antigen carriers with targeting ligands to APCs, TLR agonists or other chemical modifications may be the best pathway forward to direct an appropriate immune response for detection, elimination and prevention of future recurrence of cancer. Much work is still needed to define the antigens that should be utilized within the core of effective therapies. Evidence suggests that these antigens may have to be personalized for each patient regardless of type of cancer [116], yet considerable research is needed on how to identify optimal antigens for the most viable antitumor response. The future of cancer nanomedicine may truly be highly personalized antigen development and vaccination in conjunction with checkpoint blockade and other immunotherapies. Alternative solutions that obviate the need for a prior knowledge of the exact antigens that make up the tumor needed to reprogram the immune system may provide a bridge for solutions in the clinic until these questions are answered. Nanoparticles and related biotechnologies provide needed augmented presentation of tumor antigens for vaccine and immunotherapy treatments for cancer and undoubtedly, nanoparticle engineering for this purpose will remain an exciting focus of ongoing and future studies and an increasing focus of clinical trials and cancer treatments.

\section{Financial \& competing interests disclosure}

The authors thank Rutgers Cancer Institute of New Jersey (CINJ) and the Department of Surgery at Rutgers Robert Wood Johnson Medical School (RWJMS) for support to A Zloza and CB Chesson, which allowed the production of this review. This review was also made possible in part by support from NCI P30CA72720 to Rutgers Cancer Institute of New Jersey. CB Chesson is also supported by a fellowship from the New Jersey Commission on Cancer Research (\#DFHS17PPC009). The authors have no other relevant affiliations or financial involvement with any organization or entity with a financial interest in or financial conflict with the subject matter or materials discussed in the manuscript apart from those disclosed.

\section{Executive summary}

Nanoparticle antigen delivery platforms \& beyond

- Surface conjugation or encapsulation of whole protein or peptide antigens onto nanoparticles can facilitate antigen uptake and presentation by antigen-presenting cells leading to augment tumor antigen-specific T-cell responses.

- Virus-like particles and virosomes offer alternative platforms to nanoparticles.

- Tumor lysate and tumor-derived endosomes derived from excised tumors can provide a complete antigenic profile.

Physical determinants important for antigen presentation

- Particle size is a critical factor for determining localization within the tissue and uptake by cell type.

- Cross-presentation of antigens that are delivered by nanoparticles is important for antigen-presenting cell stimulation of T-cells responses.

- Lysosome rupture results in reactive oxygen species that enhance dendritic cell (DC) maturation leading to superior antigen presentation.

Beyond nanoparticles: ex vivo DC therapies

- DC-based treatments, although effective, are high-cost modalities and often require knowledge of specific tumor-associated antigens (TAAs).

- Nanoparticle delivery platforms that encapsulate or surface absorb TAAs, ultimately promote DC uptake, cross-presentation and maturation, while protecting TAAs from proteolytic degradation.

- The preparation of ex vivo DC vaccines typically requires the addition of adjuvants, Toll-like receptor (TLR) agonists or DC maturation ligands.

- The method of protein antigen preparation may dictate successful antigen uptake and presentation by DCs.

Beyond nanoparticles: in vivo DC targeting

- In vivo DC targeting can be used to optimize the delivery of TAAs for cancer vaccines.

- The optimal choice of DC-targeting ligands for cancer vaccines is a current subject of debate.

Codelivery of TLRs \& adjuvant combinations

- Codelivery of antigens and immunomodulatory compounds such as TLR agonists and danger-associated molecular pattern ligands provides activation signals necessary for DCs to fully mature and downstream stimulate $T$ cells and B cells.

- Nanoparticle platforms may encapsulate the antigen with surface-absorbed or covalently bound danger-associated molecular patterns, or encapsulate the antigen and adjuvant.

- Adjuvants beyond TLR agonists (including cytokines, complement pathway mediators) are also being explored for use in nanoparticle-based cancer immunotherapies. 
No writing assistance was utilized in the production of this manuscript.

\section{Open access}

This work is licensed under the Attribution-NonCommercial-NoDerivatives 4.0 Unported License. To view a copy of this license, visit http://creativecommons.org/licenses/by-nc-nd/4.0/

\section{References}

1 Lu YC, Robbins PF. Targeting neoantigens for cancer immunotherapy. Int. Immunol. 28(7), 365-370 (2016).

2 Bourla AB, Zamarin D. Immunotherapy: new strategies for the treatment of gynecologic malignancies. Oncology (Williston Park) 30(1), 59-66, 69 (2016).

3 Tang H, Qiao J, Fu YX. Immunotherapy and tumor microenvironment. Cancer Lett. 370(1), 85-90 (2016).

4 Aguilar LK, Guzik BW, Aguilar-Cordova E. Cytotoxic immunotherapy strategies for cancer: mechanisms and clinical development. J. Cell Biochem. 112(8), 1969-1977 (2011).

5 Overwijk WW, Restifo NP. Autoimmunity and the immunotherapy of cancer: targeting the "self" to destroy the "other". Crit. Rev. Immunol. 20(6), 433-450 (2000).

6 Wu AA, Drake V, Huang HS, Chiu S, Zheng L. Reprogramming the tumor microenvironment: tumor-induced immunosuppressive factors paralyze T cells. Oncoimmunology 4(7), e1016700 (2015).

$7 \mathrm{Xu} \mathrm{W}$, Cai J, Li S et al. Improving the in vivo persistence, distribution and function of cytotoxic T lymphocytes by inhibiting the tumor immunosuppressive microenvironment. Scand. J. Immunol. 78(1), 50-60 (2013).

8 Vyas JM, Van Der Veen AG, Ploegh HL. The known unknowns of antigen processing and presentation. Nat. Rev. Immunol. 8(8), 607-618 (2008).

9 Jensen PE. Recent advances in antigen processing and presentation. Nat. Immunol. 8(10), 1041-1048 (2007).

10 Overwijk WW, Wang E, Marincola FM, Rammensee HG, Restifo NP. Mining the mutanome: developing highly personalized Immunotherapies based on mutational analysis of tumors. J. Immunother. Cancer 1, 11 (2013).

11 Thomas S, Prendergast GC. Cancer vaccines: a brief overview. Methods Mol. Biol. 1403, 755-761 (2016).

12 Hofmann S, Mead A, Malinovskis A, Hardwick NR, Guinn BA. Analogue peptides for the immunotherapy of human acute myeloid leukemia. Cancer Immunol. Immunother. 64(11), 1357-1367 (2015).

13 Joshi VB, Geary SM, Gross BP, Wongrakpanich A, Norian LA, Salem AK. Tumor lysate-loaded biodegradable microparticles as cancer vaccines. Expert Rev. Vaccines 13(1), 9-15 (2014).

14 Gonzalez FE, Gleisner A, Falcon-Beas F, Osorio F, Lopez MN, Salazar-Onfray F. Tumor cell lysates as immunogenic sources for cancer vaccine design. Hum. Vaccin. Immunother. 10(11), 3261-3269 (2014).

15 Gross BP, Wongrakpanich A, Francis MB, Salem AK, Norian LA. A therapeutic microparticle-based tumor lysate vaccine reduces spontaneous metastases in murine breast cancer. AAPS J. 16(6), 1194-1203 (2014).

16 Chiang CL, Kandalaft LE, Coukos G. Adjuvants for enhancing the immunogenicity of whole tumor cell vaccines. Int. Rev. Immunol. 30(2-3), 150-182 (2011).

17 Kim J. Immune checkpoint blockade therapy for bladder cancer treatment. Investig. Clin. Urol. 57(Suppl. 1), S98-S105 (2016).

18 Vilgelm AE, Johnson DB, Richmond A. Combinatorial approach to cancer immunotherapy: strength in numbers. J. Leukocyte Biol. 100(2), 275-290 (2016)

19 Peer D, Karp JM, Hong S, Farokhzad OC, Margalit R, Langer R. Nanocarriers as an emerging platform for cancer therapy. Nat. Nano 2(12), 751-760 (2007).

20 Davis ME, Chen ZG, Shin DM. Nanoparticle therapeutics: an emerging treatment modality for cancer. Nat. Rev. Drug Discov. 7(9), 771-782 (2008).

21 Almeida JP, Lin AY, Figueroa ER, Foster AE, Drezek RA. In vivo gold nanoparticle delivery of peptide vaccine induces anti-tumor immune response in prophylactic and therapeutic tumor models. Small (Weinheim an der Bergstrasse, Germany) 11(12), 1453-1459 (2015).

22 Fifis T, Gamvrellis A, Crimeen-Irwin B et al. Size-dependent immunogenicity: therapeutic and protective properties of nano-vaccines against tumors. J. Immunol. 173(5), 3148-3154 (2004).

23 Choi B, Moon H, Hong SJ et al. Effective delivery of antigen-encapsulin nanoparticle fusions to dendritic cells leads to antigen-specific cytotoxic t cell activation and tumor rejection. ACS Nano 10(8), 7339-7350 (2016).

24 Kaluza KM, Thompson JM, Kottke TJ, Flynn Gilmer HC, Knutson DL, Vile RG. Adoptive T cell therapy promotes the emergence of genomically altered tumor escape variants. Int. J. Cancer 131(4), 844-854 (2012).

25 Kohlhapp F, Huelsmann E, Rudra J, Nabatiyan A, Zloza A. Single-step nanoparticle antigen presentation system for tumor immunotherapy. J. Immunother. Cancer 3(Suppl. 2), P319 (2015). 
26 Fang RH, Hu CM, Luk BT et al. Cancer cell membrane-coated nanoparticles for anticancer vaccination and drug delivery. Nano Lett. 14(4), 2181-2188 (2014).

27 Buonaguro L, Tagliamonte M, Tornesello ML, Buonaguro FM. Developments in virus-like particle-based vaccines for infectious diseases and cancer. Expert Rev. Vaccines 10(11), 1569-1583 (2011).

28 Pejawar-Gaddy S, Rajawat Y, Hilioti Z et al. Generation of a tumor vaccine candidate based on conjugation of a MUC1 peptide to polyionic papillomavirus virus-like particles. Cancer Immunol. Immunother. 59(11), 1685-1696 (2010).

29 Peacey M, Wilson S, Perret R et al. Virus-like particles from rabbit hemorrhagic disease virus can induce an anti-tumor response. Vaccine 26(42), 5334-5337 (2008).

30 HPV vaccine triggers regression of precancers. Cancer Discov. 5(12), 1228-1229 (2015).

31 Bryan JT, Buckland B, Hammond J, Jansen KU. Prevention of cervical cancer: journey to develop the first human papillomavirus virus-like particle vaccine and the next generation vaccine. Curr. Opin. Chem. Biol. 32, 34-47 (2016).

32 Kaneda Y. Virosome: a novel vector to enable multi-modal strategies for cancer therapy. Adv. Drug Deliv. Rev. 64(8), 730-738 (2012).

33 Abdoli A, Soleimanjahi H, Tavassoti Kheiri M, Jamali A, Mazaheri V, Abdollahpour Alitappeh M. An H1-H3 chimeric influenza virosome confers complete protection against lethal challenge with PR8 (H1N1) and X47 (H3N2) viruses in mice. Pathog. Dis. 72(3), 197-207 (2014).

34 Cusi MG, Del Vecchio MT, Terrosi C et al. Immune-reconstituted influenza virosome containing CD40L gene enhances the immunological and protective activity of a carcinoembryonic antigen anticancer vaccine. J. Immunol. 174(11), 7210-7216 (2005).

35 Wiedermann U, Wiltschke C, Jasinska J et al. A virosomal formulated Her-2/neu multi-peptide vaccine induces Her-2/neu-specific immune responses in patients with metastatic breast cancer: a Phase I study. Breast Cancer Res. Treat 119(3), 673-683 (2010).

36 Liu H, Tu Z, Feng F, Shi H, Chen K, Xu X. Virosome, a hybrid vehicle for efficient and safe drug delivery and its emerging application in cancer treatment. Acta Pharm. 65(2), 105-116 (2015).

37 Saga K, Kaneda Y. Virosome presents multimodel cancer therapy without viral replication. Biomed. Res. Int. 2013, 764706 (2013).

38 Hao S, Moyana T, Xiang J. Review: cancer immunotherapy by exosome-based vaccines. Cancer Biother. Radiopharm. 22(5), 692-703 (2007).

39 Rountree RB, Mandl SJ, Nachtwey JM et al. Exosome targeting of tumor antigens expressed by cancer vaccines can improve antigen immunogenicity and therapeutic efficacy. Cancer Res. 71(15), 5235-5244 (2011).

40 Chen W, Wang J, Shao C et al. Efficient induction of antitumor $\mathrm{T}$ cell immunity by exosomes derived from heat-shocked lymphoma cells. Eur. J. Immunol. 36(6), 1598-1607 (2006).

41 Damo M, Wilson DS, Simeoni E, Hubbell JA. TLR-3 stimulation improves anti-tumor immunity elicited by dendritic cell exosome-based vaccines in a murine model of melanoma. Sci. Rep. 5, 17622 (2015).

42 Reddy ST, Rehor A, Schmoekel HG, Hubbell JA, Swartz MA. In vivo targeting of dendritic cells in lymph nodes with poly(propylene sulfide) nanoparticles. J. Control. Release 112(1), 26-34 (2006).

43 Shukla S, Steinmetz NF. Emerging nanotechnologies for cancer immunotherapy. Exp. Biol. Med. (Maywood) 241(10), 1116-1126 (2016).

44 Wilson JT, Keller S, Manganiello MJ et al. $\mathrm{pH}$-Responsive nanoparticle vaccines for dual-delivery of antigens and immunostimulatory oligonucleotides. ACS Nano 7(5), 3912-3925 (2013).

45 Jones RA, Cheung CY, Black FE et al. Poly(2-alkylacrylic acid) polymers deliver molecules to the cytosol by pH-sensitive disruption of endosomal vesicles. Biochem. J. 372(Pt 1), 65-75 (2003).

46 Kwon YJ, Standley SM, Goodwin AP, Gillies ER, Fréchet JMJ. Directed antigen presentation using polymeric microparticulate carriers degradable at lysosomal pH for controlled immune responses. Mol. Pharm. 2(1), 83-91 (2005).

47 Standley SM, Kwon YJ, Murthy N et al. Acid-degradable particles for protein-based vaccines: enhanced survival rate for tumor-challenged mice using ovalbumin model. Bioconjug. Chem. 15(6), 1281-1288 (2004).

48 Liu Q, Chen X, Jia J et al. $\mathrm{pH}$-Responsive poly(D,L-lactic-co-glycolic acid) nanoparticles with rapid antigen release behavior promote immune response. ACS Nano 9(5), 4925-4938 (2015).

49 Sauer AM, Schlossbauer A, Ruthardt N, Cauda V, Bein T, Brauchle C. Role of endosomal escape for disulfide-based drug delivery from colloidal mesoporous silica evaluated by live-cell imaging. Nano. Lett. 10(9), 3684-3691 (2010).

50 Hirosue S, Kourtis IC, Van Der Vlies AJ, Hubbell JA, Swartz MA. Antigen delivery to dendritic cells by poly(propylene sulfide) nanoparticles with disulfide conjugated peptides: cross-presentation and T cell activation. Vaccine 28(50), 7897-7906 (2010).

51 Dingjan I, Verboogen DR, Paardekooper LM et al. Lipid peroxidation causes endosomal antigen release for cross-presentation. Sci. Rep. 6, 22064 (2016).

52 Wang C, Li P, Liu L et al. Self-adjuvanted nanovaccine for cancer immunotherapy: role of lysosomal rupture-induced ROS in MHC class I antigen presentation. Biomaterials 79, 88-100 (2016). 
53 Chen W, Yan W, Huang L. A simple but effective cancer vaccine consisting of an antigen and a cationic lipid. Cancer Immunol. Immunother. 57(4), 517-530 (2008).

54 Shen KY, Liu HY, Li HJ et al. A novel liposomal recombinant lipoimmunogen enhances anti-tumor immunity. J. Control. Release 233, 57-63 (2016).

55 Yan W, Chen W, Huang L. Reactive oxygen species play a central role in the activity of cationic liposome based cancer vaccine. J. Control. Release 130(1), 22-28 (2008).

56 Cheever MA, Higano CS. PROVENGE (Sipuleucel-T) in prostate cancer: the first FDA-approved therapeutic cancer vaccine. Clin. Cancer Res. 17(11), 3520-3526 (2011).

57 Madan RA, Gulley JL. Sipuleucel-T: harbinger of a new age of therapeutics for prostate cancer. Expert Rev. Vaccines 10(2), 141-150 (2011).

58 Mac Keon S, Ruiz MS, Gazzaniga S, Wainstok R. Dendritic cell-based vaccination in cancer: therapeutic implications emerging from murine models. Front. Immunol. 6, 243 (2015).

59 O'neill DW. Dendritic cells and T cells in immunotherapy. J. Drugs Dermatol. 9(11), 1383-1392 (2010).

60 Rainone V, Martelli C, Ottobrini L et al. Immunological characterization of whole tumour lysate-loaded dendritic cells for cancer immunotherapy. PLoS ONE 11(1), e0146622 (2016).

61 Prasad S, Cody V, Saucier-Sawyer JK et al. Polymer nanoparticles containing tumor lysates as antigen delivery vehicles for dendritic cell-based antitumor immunotherapy. Nanomedicine 7(1), 1-10 (2011).

62 Hanlon DJ, Aldo PB, Devine L et al. Enhanced stimulation of anti-ovarian cancer CD8+ T cells by dendritic cells loaded with nanoparticle encapsulated tumor antigen. Am. J. Reprod. Immunol. 65(6), 597-609 (2011).

63 Vanderlocht J, Van Elssen CH, Senden-Gijsbers BL et al. Increased tumor-specific CD8+ T cell induction by dendritic cells matured with a clinical grade TLR-agonist in combination with IFN-gamma. Int. J. Immunopathol. Pharmacol. 23(1), 35-50 (2010).

64 Gammon JM, Dold NM, Jewell CM. Improving the clinical impact of biomaterials in cancer immunotherapy. Oncotarget 7(13), 15421-15443 (2016).

65 Anderson MM, Hazen SL, Hsu FF, Heinecke JW. Human neutrophils employ the myeloperoxidase-hydrogen peroxide-chloride system to convert hydroxy-amino acids into glycolaldehyde, 2-hydroxypropanal, and acrolein. A mechanism for the generation of highly reactive alpha-hydroxy and alpha,beta-unsaturated aldehydes by phagocytes at sites of inflammation. J. Clin. Invest. 99(3), 424-432 (1997).

66 Allison ME, Fearon DT. Enhanced immunogenicity of aldehyde-bearing antigens: a possible link between innate and adaptive immunity. Eur. J. Immunol. 30(10), 2881-2887 (2000).

67 Chiang CL, Kandalaft LE, Tanyi J et al. A dendritic cell vaccine pulsed with autologous hypochlorous acid-oxidized ovarian cancer lysate primes effective broad antitumor immunity: from bench to bedside. Clin. Cancer Res. 19(17), 4801-4815 (2013).

68 Jie X, Hua L, Jiang W, Feng F, Feng G, Hua Z. Clinical application of a dendritic cell vaccine raised against heat-shocked glioblastoma. Cell Biochem. Biophys. 62(1), 91-99 (2012).

69 Vandenberk L, Belmans J, Van Woensel M, Riva M, Van Gool SW. Exploiting the immunogenic potential of cancer cells for improved dendritic cell vaccines. Front. Immunol. 6, 663 (2015).

70 Fan Y, Moon JJ. Nanoparticle drug delivery systems designed to improve cancer vaccines and immunotherapy. Vaccines (Basel) 3(3), 662-685 (2015).

71 Thomas SN, Vokali E, Lund AW, Hubbell JA, Swartz MA. Targeting the tumor-draining lymph node with adjuvanted nanoparticles reshapes the anti-tumor immune response. Biomaterials 35(2), 814-824 (2014).

72 Strioga MM, Felzmann T, Powell DJ Jr et al. Therapeutic dendritic cell-based cancer vaccines: the state of the art. Crit. Rev. Immunol. 33(6), 489-547 (2013).

73 Linch SN, Redmond WL. How do I steer this thing? Using dendritic cell targeted vaccination to more effectively guide the antitumor immune response with combination immunotherapy. J. Immunother. Cancer 4, 31 (2016).

74 Macri C, Dumont C, Johnston AP, Mintern JD. Targeting dendritic cells: a promising strategy to improve vaccine effectiveness. Clin. Transl. Immunol. 5(3), e66 (2016).

75 Yin W, Gorvel L, Zurawski S et al. Functional specialty of CD40 and dendritic cell surface lectins for exogenous antigen presentation to CD8(+) and CD4(+) T cells. EBioMedicine 5, 46-58 (2016).

76 Lehmann CH, Heger L, Heidkamp GF et al. Direct delivery of antigens to dendritic cells via antibodies specific for endocytic receptors as a promising strategy for future therapies. Vaccines (Basel) 4(2), 8 (2016).

77 Kokate RA, Chaudhary P, Sun X et al. Rationalizing the use of functionalized poly-lactic-co-glycolic acid nanoparticles for dendritic cell-based targeted anticancer therapy. Nanomedicine (Lond.) 11(5), 479-494 (2016).

78 Wang Q, Cao W, Yang ZG, Zhao GF. DC targeting DNA vaccines induce protective and therapeutic antitumor immunity in mice. Int J. Clin. Exp. Med. 8(10), 17565-17577 (2015).

79 Tacken PJ, Ginter W, Berod L et al. Targeting DC-SIGN via its neck region leads to prolonged antigen residence in early endosomes, delayed lysosomal degradation, and cross-presentation. Blood 118(15), 4111-4119 (2011). 
80 Dudziak D, Kamphorst AO, Heidkamp GF et al. Differential antigen processing by dendritic cell subsets in vivo. Science 315(5808), 107-111 (2007).

81 Singh SK, Stephani J, Schaefer M et al. Targeting glycan modified OVA to murine DC-SIGN transgenic dendritic cells enhances MHC class I and II presentation. Mol. Immunol. 47(2-3), 164-174 (2009).

82 Van Kooyk Y, Unger WW, Fehres CM, Kalay H, Garcia-Vallejo JJ. Glycan-based DC-SIGN targeting vaccines to enhance antigen cross-presentation. Mol. Immunol. 55(2), 143-145 (2013).

83 Kim WG, Choi B, Yang HJ et al. Covalent conjugation of small-molecule adjuvants to nanoparticles induces robust cytotoxic $\mathrm{T}$ cell responses via DC activation. Bioconjug. Chem. 27(9), 2007-2013 (2016).

84 Dajon M, Iribarren K, Cremer I. Toll-like receptor stimulation in cancer: a pro- and anti-tumor double-edged sword. Immunobiology 222(1), 89-100 (2016).

85 Kaczanowska S, Joseph AM, Davila E. TLR agonists: our best frenemy in cancer immunotherapy. J. Leuk. Biol. 93(6), 847-863 (2013).

86 Temizoz B, Kuroda E, Ishii KJ. Vaccine adjuvants as potential cancer immunotherapeutics. Int. Immunol. 28(7), 329-338 (2016).

87 Zhou Z, Yu X, Zhang J, Tian Z, Zhang C. TLR7/8 agonists promote NK-DC cross-talk to enhance NK cell anti-tumor effects in hepatocellular carcinoma. Cancer Lett. 369(2), 298-306 (2015).

88 Wang C, Zhou Q, Wang X et al. The TLR7 agonist induces tumor regression both by promoting CD4(+)T cells proliferation and by reversing T regulatory cell-mediated suppression via dendritic cells. Oncotarget 6(3), 1779-1789 (2015).

89 Nakamura T, Miyabe H, Hyodo M, Sato Y, Hayakawa Y, Harashima H. Liposomes loaded with a STING pathway ligand, cyclic di-GMP, enhance cancer immunotherapy against metastatic melanoma. J. Control. Release 216, 149-157 (2015).

90 Miyabe H, Hyodo M, Nakamura T, Sato Y, Hayakawa Y, Harashima H. A new adjuvant delivery system 'cyclic di-GMP/YSK05 liposome' for cancer immunotherapy. J. Control. Release 184, 20-27 (2014).

91 Yu L, Wang L, Chen S. Dual character of Toll-like receptor signaling: pro-tumorigenic effects and anti-tumor functions. Biochim. Biophys. Acta 1835(2), 144-154 (2013).

92 Ghinnagow R, Cruz LJ, Macho-Fernandez E, Faveeuw C, Trottein F. Enhancement of adjuvant functions of natural killer T cells using nanovector delivery systems: application in anticancer immune therapy. Front. Immunol. 8, 879 (2017).

93 Neumann S, Young K, Compton B, Anderson R, Painter G, Hook S. Synthetic TRP2 long-peptide and $\alpha$-galactosylceramide formulated into cationic liposomes elicit CD8+ T-cell responses and prevent tumour progression. Vaccine 33(43), 5838-5844 (2015).

94 Dong T, Yi T, Yang M et al. Co-operation of alpha-galactosylceramide-loaded tumour cells and TLR9 agonists induce potent anti-tumour responses in a murine colon cancer model. Biochem. J. 473(1), 7-19 (2016).

95 Hamdy S, Molavi O, Ma Z et al. Co-delivery of cancer-associated antigen and Toll-like receptor 4 ligand in PLGA nanoparticles induces potent CD8+ T cell-mediated anti-tumor immunity. Vaccine 26(39), 5046-5057 (2008).

96 Li WA, Lu BY, Gu L, Choi Y, Kim J, Mooney DJ. The effect of surface modification of mesoporous silica micro-rod scaffold on immune cell activation and infiltration. Biomaterials 83, 249-256 (2016).

97 Kim J, Li WA, Choi Y et al. Injectable, spontaneously assembling, inorganic scaffolds modulate immune cells in vivo and increase vaccine efficacy. Nat. Biotechnol. 33(1), 64-72 (2015).

98 Muraoka D, Harada N, Hayashi T et al. Nanogel-based immunologically stealth vaccine targets macrophages in the medulla of lymph node and induces potent antitumor immunity. ACS Nano 8(9), 9209-9218 (2014).

99 Forghani P, Waller EK. Poly (I: C) modulates the immunosuppressive activity of myeloid-derived suppressor cells in a murine model of breast cancer. Breast Cancer Res. Treat 153(1), 21-30 (2015).

100 Chang LS, Leng CH, Yeh YC et al. Toll-like receptor 9 agonist enhances anti-tumor immunity and inhibits tumor-associated immunosuppressive cells numbers in a mouse cervical cancer model following recombinant lipoprotein therapy. Mol. Cancer 13, 60 (2014).

101 Heo MB, Cho MY, Lim YT. Polymer nanoparticles for enhanced immune response: combined delivery of tumor antigen and small interference RNA for immunosuppressive gene to dendritic cells. Acta Biomater. 10(5), 2169-2176 (2014).

102 Ballester M, Jeanbart L, De Titta A et al. Nanoparticle conjugation enhances the immunomodulatory effects of intranasally delivered CpG in house dust mite-allergic mice. Sci. Rep. 5, 14274 (2015).

103 Saleh T, Shojaosadati SA. Multifunctional nanoparticles for cancer immunotherapy. Hum. Vaccin. Immunother. 12(7), 1863-1875 (2016).

104 Goldinger SM, Dummer R, Baumgaertner P et al. Nano-particle vaccination combined with TLR-7 and -9 ligands triggers memory and effector CD8(+) T-cell responses in melanoma patients. Eur. J. Immunol. 42(11), 3049-3061 (2012).

105 Rosalia RA, Cruz LJ, Van Duikeren S et al. CD40-targeted dendritic cell delivery of PLGA-nanoparticle vaccines induce potent anti-tumor responses. Biomaterials 40, 88-97 (2015).

106 Srivastava AK, Yolcu ES, Dinc G, Sharma RK, Shirwan H. SA-4-1BBL/MPL as a novel immune adjuvant platform to combat cancer. Oncoimmunology 5(1), e1064580 (2016). 
107 Sharma RK, Yolcu ES, Srivastava AK, Shirwan H. CD4+ T cells play a critical role in the generation of primary and memory antitumor immune responses elicited by SA-4-1BBL and TAA-based vaccines in mouse tumor models. PLoS ONE 8(9), e73145 (2013).

108 Sharma RK, Schabowsky RH, Srivastava AK et al. 4-1BB ligand as an effective multifunctional immunomodulator and antigen delivery vehicle for the development of therapeutic cancer vaccines. Cancer Res. 70(10), 3945-3954 (2010).

109 Chatterjee S, Crozet L, Damotte D et al. TLR7 promotes tumor progression, chemotherapy resistance, and poor clinical outcomes in non-small cell lung cancer. Cancer Res. 74(18), 5008-5018 (2014).

110 Cherfils-Vicini J, Platonova S, Gillard M et al. Triggering of TLR7 and TLR8 expressed by human lung cancer cells induces cell survival and chemoresistance. J. Clin. Invest. 120(4), 1285-1297 (2010).

111 Floreani AA, Gunselman SJ, Heires AJ, Hauke RJ, Tarantolo S, Jackson JD. Novel C5a agonist-based dendritic cell vaccine in a murine model of melanoma. Cell Cycle 6(22), 2835-2839 (2007).

112 Rudilla F, Fayolle C, Casares N et al. Combination of a TLR4 ligand and anaphylatoxin C5a for the induction of antigen-specific cytotoxic T cell responses. Vaccine 30(18), 2848-2858 (2012).

113 Kraynyak KA, Kutzler MA, Cisper NJ et al. Plasmid-encoded interleukin-15 receptor alpha enhances specific immune responses induced by a DNA vaccine in vivo. Hum. Gene Ther. 20(10), 1143-1156 (2009).

114 Hong E, Usiskin IM, Bergamaschi C et al. Configuration-dependent presentation of multivalent IL-15:IL-15Ralpha enhances the antigen-specific T cell response and anti-tumor immunity. J. Biol. Chem. 291(17), 8931-8950 (2016).

115 Overwijk WW, De Visser KE, Tirion FH et al. Immunological and antitumor effects of IL-23 as a cancer vaccine adjuvant. J. Immunol. 176(9), 5213-5222 (2006).

116 Hartmaier RJ, Charo J, Fabrizio D et al. Genomic analysis of 63,220 tumors reveals insights into tumor uniqueness and targeted cancer immunotherapy strategies. Genome Med. 9(1), 16 (2017) 